\title{
Minimal hepatic encephalopathy - diagnosis and treatment
}

\author{
Wojciech Pisarek \\ Department of Gastroenterology and Hepatology, Wroclaw Medical University, Wroclaw, Poland
}

Gastroenterology Rev 2021; 16 (4): 311-317

DOI: https://doi.org/10.5114/pg.2021.111389

Key words: minimal hepatic encephalopathy, psychometric tests, neurophysiological test, chronic liver diseases, rifaximin $\alpha$.

Address for correspondence: Wojciech Pisarek MD, Department of Gastroenterology and Hepatology, Wroclaw Medical University, 50-556 Wroclaw, Poland, phone: +48 883966694, e-mail: lek.pisarekwojciech@gmail.com

\begin{abstract}
Hepatic encephalopathy is a dysfunction of the central nervous system caused by chronic and acute liver disease. It presents a wide spectrum of symptoms from undetectable in a standard clinical examination to hepatic coma. The mildest form of hepatic encephalopathy is minimal hepatic encephalopathy. It significantly influences the quality of life, prognosis, and the incidence of complications. A wide range of psychometric and neurophysiological tests are used in the diagnostics. Treatment is based on the same principles as in overt encephalopathy. The most commonly used drugs include rifaximin, ornithine aspartate, and LOLA.
\end{abstract}

\section{Introduction}

Hepatic encephalopathy is one of the most important complications of both acute and chronic liver diseases and portal-systemic bypassing. It is a potentially reversible complex of neurological, psychiatric, and musculoskeletal disorders. Patients with hepatic encephalopathy often have significant deterioration of cognitive functions, disturbances in sleep and wake rhythm, memory disorders, prolonged reaction time to stimuli [1], increased risk of falls, more frequent road traffic hazards, and deterioration of health-related quality of life (HRQoL) [2].

According to the current recommendations of the European Association for the Study of the Liver (EASL) and American Association for the Study of Liver Diseases (AASLD) [3], the classification of hepatic encephalopathy should include 4 specific factors. One of them is the severity of the clinical manifestation. There is a classification according to West Heaven Criteria (WHC), which divides encephalopathy into minimal hepatic encephalopathy and 4 degrees of overt clinical encephalopathy, as well as a classification according to International Society for Hepatic Encephalopathy and Nitrogen Metabolism (ISHEN), which divides encephalopathy into overt and covert encephalopathy. ISHEN covert encephalopathy includes minimal hepatic encephalopathy and WHC first-degree overt encepha- lopathy. The other 3 factors are association with the underlying disease, disease duration, and the presence of triggers

Minimal hepatic encephalopathy (MHE) is the mildest form of hepatic encephalopathy. It is characterized by discrete motor and cognitive impairment, which is impossible to detect in a standard physical examination. According to the definition given in the AASLD guidelines [3], MHE is the presence of brain dysfunction detected in diagnostic tests or based on clinical signs in patients without orientation disorders and hand tremor (asterixis). The prevalence of MHE in chronic liver diseases is estimated at $30-80 \%$ of patients with chronic liver disease [4]. This large dispersion results from the lack of standardized research methods and the specificity of the studied populations. Despite the lack of obvious clinical symptoms, MHE significantly affects the daily life of patients. Patients with MHE have significantly reduced health-related quality of life [5]. Its occurrence increases the risk of overt hepatic encephalopathy and worsens the prognosis [6]. MHE also impairs the performance of complex tasks such as driving. It has been proven that patients with MHE violate traffic regulations and cause accidents more often. They also perform worse on driving simulators.

Due to the lack of guidelines for the diagnosis and treatment of MHE, despite its significant impact on the 
lives of patients, this problem still receives insufficient attention.

A 2007 study conducted in the USA showed that only $50 \%$ of physicians consider the possibility of $\mathrm{MHE}$ in patients with chronic liver disease.

The socioeconomic aspect of MHE is also important. The costs incurred by the health care system due to the lack of early detection and treatment of MHE, which would prevent complications and the development of overt hepatic encephalopathy, are very high [7].

\section{Diagnostics}

Minimal hepatic encephalopathy is still an underdiagnosed problem. This is due to the small number of multicentre randomized trials, and the lack of clear guidelines for recommended diagnostic methods and of qualified personnel.

According to the AASLD/EASL guidelines, MHE testing should be performed in patients who can benefit most from it [3], i.e. patients with reduced quality of life due to liver diseases or people employed in positions related to public safety. It should also be remembered that early detection of MHE can reduce the burden on the health care system and improve the quality of life of patients.

MHE tests (Table I) can be divided into several groups: psychometric, neurophysiological, neuroimaging, and laboratory tests. Not all are currently recommended. AASLD/EASL recommends at least 2 different tests in multicentre clinical trials. One of them should be the psychometric hepatic encephalopathy test score (PHES test), supplemented by one of the computer psychometric tests (continuous reaction test (CRT), inhibitory control test (ICT), SCAN Test, Stroop Test) or neurophysiological tests (critical flicker frequency (CFF) or electroencephalogram (EEG)). It seems that a similar strategy can also be used in everyday clinical practice. Diagnostics should be carried out by experienced personnel; it

Table I. MHE tests

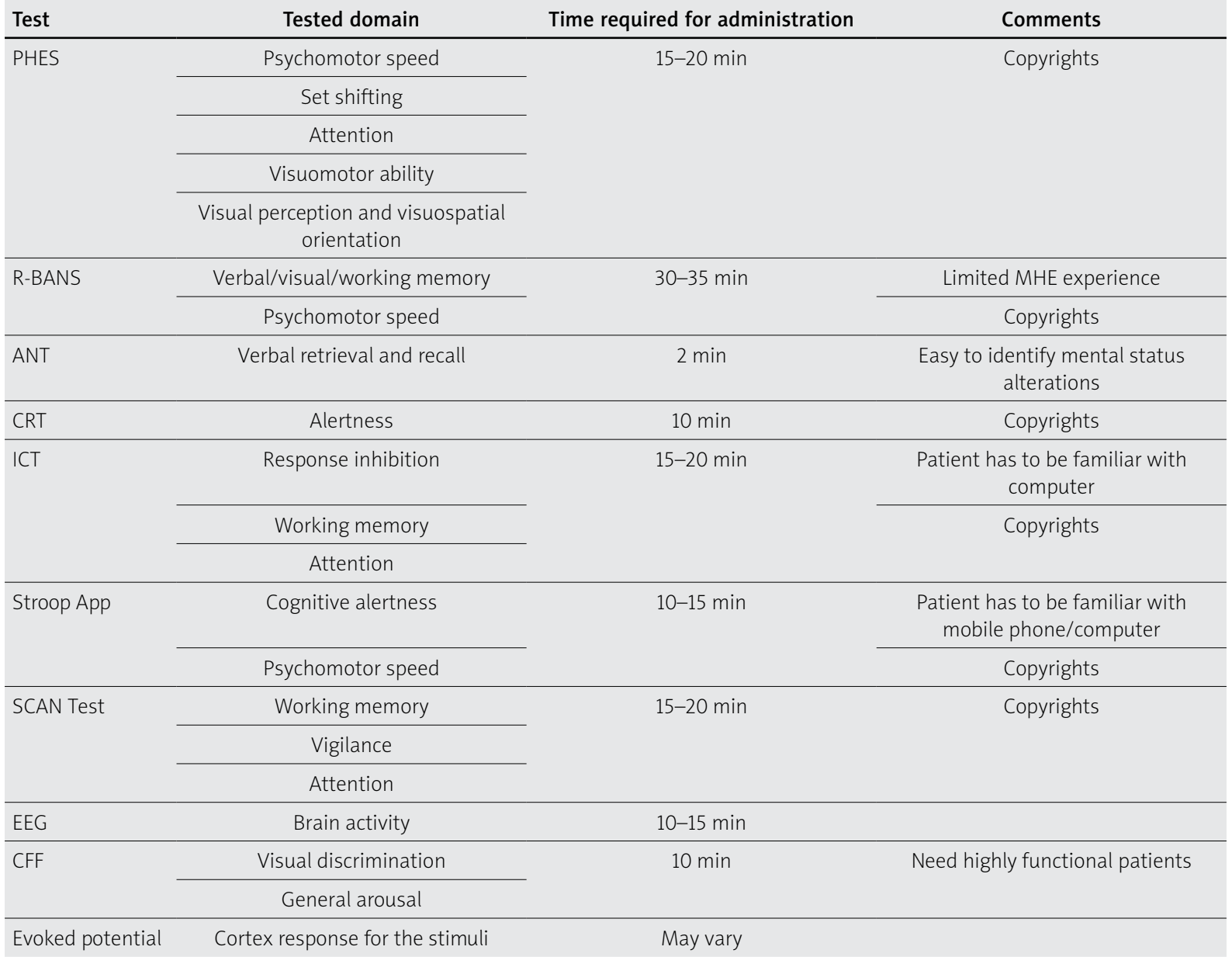

Modified from: Kappus MR, Bajaj JS. Covert hepatic encephalopathy: not as minimal as you might think. Clin Gastroenterol Hepatol 2012; 10: 1208-19. PHES - psychometric hepatic encephalopathy score, R-BANS - repeatable battery for assessment of neuropsychological status, CRT - continuous reaction time, ICT - inhibitory control test, EEG - electroencephalogram, CFF - critical flicker frequency. 
is also important to validate the tests used in a given population. Before using any of the tests, the presence of other factors influencing the central nervous system should be excluded: organic brain diseases, mental disorders, metabolic diseases, or dementia. The MMSE test (Mini-Mental State Examination) seems to be beneficial as a screening test before starting MHE diagnostics.

\section{Psychometric tests PHES test}

The PHES (psychometric hepatic encephalopathy score) test is considered the gold standard in the diagnosis of MHE. It consists of 5 paper-and-pencil test sheets: number connection test A and B (NCT-A and NCT-B), serial dotting test (SDT), digit symbol test (DST), and line tracing test (LTT). These tests are designed to assess concentration, attention, memory, speed, and motor and visual accuracy [8]. This test suite is designed to detect minimal hepatic encephalopathy [1] and has been validated in populations of many countries, including Poland [9]. The PHES test can also be used as a prognostic tool for overt hepatic encephalopathy and the survival of patients with MHE, and it may also be helpful in identifying patients at higher risk of falls. A trained worker takes approximately $20 \mathrm{~min}$ to perform these tests, which may limit their use in ambulatory conditions. The results may be influenced by the patient's level of education and age [10]. A learning effect was also observed, which disappeared when the test was performed less frequently than every 6 months.

\section{R-BANS test}

This is another paper-and-pencil test initially designed to assess other neurocognitive disorders (e.g. in Alzheimer's disease). It is a test well validated in the US population for the diagnosis of cognitive disorders. Nowadays there are no standards for populations of other countries, which means that its clinical use requires appropriate standardization. ISHEN recommends performing the RBANS test together with the PHES test. However, there are still no studies that would assess the sensitivity and specificity of this test in patients with MHE.

\section{Animal naming test (ANT)}

This is an extremely simple test that allows the patient to list as many species of animals as possible within $1 \mathrm{~min}$. Clinical data indicates that this test can be used as a screening test to identify patients who require testing for MHE [11]. The undoubted advantage of this test is that it can be performed during a telephone conversation, which allows for regular ambulatory inspection of patients.

\section{Continuous response time (CRT) test}

A relatively new method for the detection of $\mathrm{MHE}$. This test is based on a response to auditory stimuli. For $10 \mathrm{~min}$, the patient is subjected to 150 auditory stimuli of varying frequency with the task of pressing a button as soon as possible after hearing the stimulus. The result is presented in the form of an indicator - the CRT index, which assesses the stability of response time in individual trials. This test allows the differentiation of metabolic and organic damage to the CNS. The lack of a learning effect is also important [12].

\section{Inhibitory Control Test (ICT)}

This is a computerized test consisting of the patient finding a specific combination of letters (the letter $Y$ immediately after the letter $X$ or vice versa) among different letters appearing at a time interval of $500 \mathrm{~ms}$ [13]. The test also includes baits ( $X$ following $X$, or $Y$ following $Y)$. The results are analysed automatically in a computer program. This test was initially used to assess cognitive deficits in patients with schizophrenia and traumatic brain injury. Research results indicate the possibility of using ICT as an alternative to PHES in detecting MHE [14]. An important advantage of this test is also free access to the software.

\section{EncephalApp (Stroop App)}

One of the latest diagnostic tests comes in the form of an application for mobile devices. The application has been available since 2013 [15], currently for both iOS and Android devices. The application uses the so-called Stroop effect to assess psychomotor speed and cognitive alertness. The patient's task is to identify the colour of the stimulus presented on the screen. The complete examination consists of 3 stages. In the first, the patient determines the colour in which a random word is displayed on the screen. In the second, the patient should name the colour of the word that is the colour definition, which is in line with the name. In the third step, the colour name and the colour of the word do not match. The test result is available immediately after the test is performed. Studies in the US population have shown good sensitivity and high specificity of the test and effectiveness in assessing the risk of progression to overt hepatic encephalopathy [16].

The undoubted advantage of this method is its availability; the application is free, and the test can be performed in an ambulatory or home setting. The test is easy to apply in a short time. However, the application requires standardization. 


\section{SCAN-test}

This is a computerized test assessing the speed and accuracy of recognizing a given stimulus. By pressing the appropriate button, the patient confirms or denies the presence of a given digit in a set presented earlier. The program measures the reaction time and the number of mistakes made. The test has been validated in the Italian population [17], and its prognostic value has also been confirmed in patients with cirrhosis of the liver. There is no validation of the test in the Polish population.

\section{Neurophysiological methods EEG}

Electroencephalography has been used in the diagnosis of hepatic encephalopathy for a long time. The Persons-Smith classification, currently modified by Amodio [17], is used to assess EEG disturbances in the course of hepatic encephalopathy. Cortical brain activity is assessed. It is an available examination that does not require the patient's cooperation, but its results may be influenced by metabolic disorders, other CNS diseases, and the drugs used. Due to the dependence of the result on the evaluating investigator, it is recommended to use spectral, computer-assisted EEG analysis [18]. In some studies, EEG abnormalities were found in patients with MHE in as many as $85 \%$ of patients without clinical symptoms of encephalopathy. However, the use of EEG as the primary diagnostic method in MHE is still widely discussed. The need to cooperate with an experienced physician who interprets the EEG record is also emphasized, as well as a relatively large amount of time needed to perform and describe the test.

\section{Critical Flicker Frequency (CFF) Test}

This test was initially used in otolaryngology to diagnose changes in the optic nerve. It has been used in the diagnosis of MHE since 2002 [19]. The test consists of stimulating the patient with light stimuli of decreasing frequency (from 60 to $25 \mathrm{~Hz}$ ). The patient's task is to press the appropriate button when the visible light changes from constant to blinking. The test result is averaged over 8 samples. A meta-analysis of 9 studies [20] proved that this test has high specificity and moderate sensitivity. A significant advantage of the test is the easy understanding of the test rules by patients and the lack of a learning effect. The test application is limited by the use of expensive equipment, and the need for binocular vision and correct colour vision.

\section{Evoked potential}

This test records the brain's bioelectrical response to visual, auditory, or somatosensory stimuli. The P300 auditory potentials are the most commonly used. However, there are currently no clear guidelines and recommendations for their use, which limits their use in clinical practice. The problem is also the high price of the necessary equipment.

\section{Neuroimaging methods}

Imaging diagnostics of the brain has made a very large contribution to research into the pathophysiology of hepatic encephalopathy. It also allows us to exclude other causes of damage to the central nervous system. Despite many studies, no diagnostic protocols for MHE using neuroimaging methods have been developed so far.

\section{Computed tomography}

Computed tomography is currently used mainly as an auxiliary examination to rule out organic brain diseases. A 2006 study [21] described the presence of cortical atrophy of the frontal region and a slight degree of cerebral oedema in MHE patients.

\section{Magnetic resonance imaging}

Magnetic resonance imaging is currently used only in clinical trials and to rule out organic CNS diseases.

\section{Positron emission tomography}

Studies using 18-fluorodeoxyglucose in patients with MHE have shown reduced marker metabolism in the anterior cingulate cortex, in the occipital-parietal cortex, and in the temporal lobe. These are the areas of the cortex that are responsible for associative abilities. At present, however, there are insufficient data necessary to consider PET a recommended diagnostic method

\section{Treatment}

Due to the insufficient number of multi-centre studies, it is currently not recommended to routinely treat every patient with MHE. However, the AASLD and EASL guidelines suggest that each case should be treated individually [3]. Therefore, it is possible to introduce pharmacotherapy according to the guidelines used in the treatment of overt hepatic encephalopathy. When planning treatment, the physician should take into account the lifestyle, type of work, and the patient's well-being and quality of life.

\section{Rifaximin}

Rifaximin $\alpha$ is an orally administered antibiotic that is not absorbed from the gastrointestinal tract. Its chemical structure is similar to that of rifampicin; it inhibits the synthesis of RNA and proteins in bacte- 
rial cells. It also has a eubiotic effect - it modifies the composition of the intestinal microflora and thus reduces the intestinal production of ammonia and other harmful substances produced by anaerobic bacteria (including mercaptans, GABA, short- and medium-chain fatty acids). The beneficial effect of rifaximin in patients with MHE has been proven. A study by Bajaj et al. [22] showed that 8-week rifaximin therapy significantly improves the results obtained by patients with $\mathrm{MHE}$ during driving simulator tests. Sidhu's research [23] showed a significant advantage of rifaximin over placebo in the treatment of MHE patients, and improved health-related quality of life in patients.

\section{Lactulose}

Lactulose is a non-absorbable disaccharide with a multidirectional, beneficial effect in the gastrointestinal tract. It accelerates intestinal transit, and thus reduces the amount of toxins absorbed from the intestine. Additionally, non-absorbable disaccharides stimulate the development of physiological bacterial flora and reduce the amount of potentially harmful bacteria. Lactulose also helps to lower ammonia levels by acidifying the environment, which facilitates its conversion to ammonium ions. In a 2012 study, Sharma et al. showed that lactulose was significantly more effective than placebo in improving the results of psychometric tests in patients with MHE [24]. A meta-analysis of 9 studies [25] showed that lactulose significantly reduces serum ammonia levels and has a positive effect on health-related quality of life (HRQoL) in patients with MHE. A recently published, multicentre study in the Chinese population has demonstrated the beneficial effects of lactulose on cognitive functions, quality of life, and the composition of the gut microflora in MHE patients [26]. A limitation in long-term use of lactulose is the relatively high frequency of side effects such as flatulence, diarrhoea, and pain in the abdominal cavity

\section{L-ornithine L-aspartate (LOLA)}

LOLA is a salt of the amino acids ornithine and asparagine, which is easily dissociated into single amino acids. L-ornithine and L-asparagine stimulate the synthesis of glutamate in the liver, brain, and muscles. L-ornithine is also involved in the hepatocyte urea cycle, influencing the conversion of toxic ammonia into urea. In a meta-analysis from 2018. Goh et al. [27], analysing 9 clinical trials, showed the effectiveness of both intravenous and oral LOLA administration in patients with MHE. Similarly, a meta-analysis of 27 clinical trials [28] showed the effectiveness of orally administered LOLA in reducing serum ammonia levels and improving psychomotor test scores in MHE patients.

\section{Branched chain amino acids (BCAA)}

Exogenous branched chain amino acids (leucine, isoleucine, valine) have long attracted the attention of clinicians as a possible form of ECT therapy. They are currently believed to act by positively affecting serum ammonia levels by favouring skeletal muscle. In skeletal muscle, ammonia is converted into glutamine. An excessive concentration of ammonia in the serum impairs this mechanism. The use of BCAAs allows this mechanism to be counteracted. The meta-analysis of Thumburu et al. assessed the effectiveness of MHE treatment with the use of BCAAs [28]. The results showed a beneficial effect of BCAAs on serum ammonia levels.

\section{Other methods of treatment \\ L-carnitine}

This is an organic chemical compound with a betaine structure, which is a metabolite of lysine. In the human body, it plays a role in the transport of fatty acids from the cytoplasm to the mitochondria. A randomized study involving 67 patients with MHE has shown a beneficial effect of L-carnitine on the well-being and energy level of patients [29].

\section{Probiotics}

Due to the influence of the intestinal microflora on the production of ammonia, probiotics have been of interest to clinicians for a long time. In a 2015 meta-analysis, Zhao et al. analysed 9 randomized clinical trials [30]. It has been shown that the use of probiotics improves the condition of patients with MHE, reduces the risk of progression to overt hepatic encephalopathy, and may also reduce the risk of acute complications.

Due to the lack of clear guidelines for the treatment of MHE, the decision to apply the treatment and the choice of the drug rests with the attending physician. Further studies on the effectiveness of treatment methods are necessary to develop guidelines and patterns of management for patients with MHE.

\section{Summary}

Due to the negative impact of MHE on quality of life, prognosis, and burden on the healthcare system, its detection and treatment should be one of the priorities for clinicians dealing with patients with chronic liver diseases.

The limitation is the lack of clearly formulated and up-to-date guidelines that would allow a simple, cheap, and repeatable control of this group of patients. An additional problem is the lack of large multicentre clinical trials that would help in updating the guidelines and selecting diagnostic methods. 
The close cooperation of gastroenterologists, neurologists, and psychiatrists in the diagnostic process also seems to be important. Currently, the assessment of the cognitive functions of patients with chronic liver diseases is too rare, usually only in specialized centres. It is extremely important to educate primary care physicians in the context of the use of simple paper-and-pencil diagnostic methods as well as periodic evaluation of patients with MHE who wait too long for specialist consultations.

\section{Conflict of interest}

The author declares no conflict of interest.

\section{References}

1. Weissenborn K, Ennen JC, Schomerus H, et al. Neuropsychological characterization of hepatic encephalopathy. J Hepatol 2001; 34: 768-73.

2. Schomerus H, Hamster W. Quality of life in cirrhotics with minimal hepatic encephalopathy. Metabolic Brain Dis 2001; 16: 37-41.

3. Vilstrup H, Amodio P, Bajaj J, et al. Hepatic encephalopathy in chronic liver disease: 2014 Practice Guideline by the American Association for the Study Of Liver Diseases and the European Association for the Study of the Liver. Hepatology 2014; 60: 715-35.

4. Bajaj J. Minimal hepatic encephalopathy matters in daily life. World J Gastroenterol 2008; 14: 3609-15.

5. Mina A, Moran S, Ortiz-Olvera N, et al. Prevalence of minimal hepatic encephalopathy and quality of life in patients with decompensated cirrhosis. Hepatol Res 2014; 44: 92-9.

6. Romero-Gómez M, Montagnese S, Jalan R. Hepatic encephalopathy in patients with acute decompensation of cirrhosis and acute-on-chronic liver failure. J Hepatol 2015; 62: 437-47.

7. Bajaj JS, Wade JB, Gibson DP, et al. The multi-dimensional burden of cirrhosis and hepatic encephalopathy on patients and caregivers. Am J Gastroenterol 2011; 106: 1646-53.

8. Luo M, Ma P, Li L, et al. Advances in psychometric tests for screening minimal hepatic encephalopathy: from paper-and-pencil to computer-aided assessment. Turk J Gastroenterol 2019; 30: 398-407.

9. Wunsch E, Koziarska D, Kotarska K, et al. Normalization of the psychometric hepatic encephalopathy score in Polish population. A prospective, quantified electroencephalography study. Liver Int 2013; 33: 1332-40

10. Nabi E, Thacker LR, Wade JB, et al. Diagnosis of covert hepatic encephalopathy without specialized tests. Clin Gastroenterol Hepatol 2014; 12: 1384-9.

11. Campagna F, Montagnese S, Ridola L, et al. The animal naming test: an easy tool for the assessment of hepatic encephalopathy. Hepatology 2017; 66: 198-208.

12. Lauridsen MM, Thiele M, Kimer N, et al. The continuous reaction times method for diagnosing, grading, and monitoring minimal/covert hepatic encephalopathy. Metabolic Brain Disease 2013; 28: 231-4.
13. Bajaj JS, Hafeezullah M, Franco J, et al. Inhibitory control test for the diagnosis of minimal hepatic encephalopathy. Gastroenterology 2008; 135: 1591-600.

14. Gupta D, Ingle M, Shah K, et al. Prospective comparative study of inhibitory control test and psychometric hepatic encephalopathy score for diagnosis and prognosis of minimal hepatic encephalopathy in cirrhotic patients in the Indian subcontinent. J Dig Dis 2015; 16: 400-7.

15. Bajaj JS, Thacker LR, Heuman DM, et al. The stroop smartphone application is a short and valid method to screen for minimal hepatic encephalopathy. Hepatology 2013; 58: 1122-32.

16. Allampati S, Duarte-Rojo A, Thacker LR, et al. Diagnosis of minimal hepatic encephalopathy using Stroop EncephalApp: a multicenter US-Based, norm-based study. Am J Gastroenterol 2016; 111: 78-86.

17. Amodio P, Del Piccolo F, Marchetti P, et al. Clinical features and survival of cirrhotic patients with subclinical cognitive alterations detected by the number connection test and computerized psychometric tests. Hepatology 1999; 29: 1662-7.

18. Guerit JM, Amantini A, Fischer C, et al. Neurophysiological investigations of hepatic encephalopathy: ISHEN practice guidelines. Liver Int 2009; 29: 789-96.

19. Häussinger D, Wettstein M, Kircheis $G$, et al. Critical flicker frequency for quantification of low-grade hepatic encephalopathy. Hepatology 2002; 35: 357-66.

20. Torlot FJ, McPhail MJW, Taylor-Robinson SD. Meta-analysis: the diagnostic accuracy of critical flicker frequency in minimal hepatic encephalopathy. Alimentary Pharmacol Ther 2013; 37: 527-36.

21. Quero Guillén JC, Herrerías Gutiérrez JM. Diagnostic methods in hepatic encephalopathy. Clin Chim Acta 2006; 365: 1-8.

22. Bajaj JS, Heuman DM, Wade JB, et al. Rifaximin improves driving simulator performance in a randomized trial of patients with minimal hepatic encephalopathy. Gastroenterology 2011; 140: 478-87.

23. Sidhu SS, Goyal O, Mishra BP, et al. Rifaximin improves psychometric performance and health-related quality of life in patients with minimal hepatic encephalopathy (the RIME trial). Am J Gastroenterol 2011; 106: 307-16.

24. Sharma P, Sharma BC, Agrawal A, et al. Primary prophylaxis of overt hepatic encephalopathy in patients with cirrhosis: an open labeled randomized controlled trial of lactulose versus no lactulose. J Gastroenterol Hepatol 2012; 27: 1329-35.

25. Luo M, Li L, Lu CZ, et al. Clinical efficacy and safety of lactulose for minimal hepatic encephalopathy: a meta-analysis. Eur J Gastroenterol Hepatol 2011; 23: 1250-7.

26. Wang JY, Bajaj JS, Wang JB, et al. Lactulose improves cognition, quality of life, and gut microbiota in minimal hepatic encephalopathy: a multicenter, randomized controlled trial. J Dig Dis 2019; 20: 547-56.

27. Goh ET, Stokes CS, Sidhu SS, et al. L-ornithine L-aspartate for prevention and treatment of hepatic encephalopathy in people with cirrhosis. Cochrane Database Syst Rev 2018; 5: CD012410.

28. Thumburu KK, Dhiman RK, Chopra M, et al. Comparative effectiveness of different pharmacological interventions for the treatment of minimal hepatic encephalopathy: a systematic 
review with network meta-analysis. J Clin Exp Hepatol 2017;

7: 6-7.

29. Malaguarnera M, Gargante MP, Cristaldi E, et al. Acetyl I-carnitine (ALC) treatment in elderly patients with fatigue. Arch Gerontol Geriatrics 2008; 46: 181-90.

30. Zhao LN, Yu T, Lan SY, et al. Probiotics can improve the clinical outcomes of hepatic encephalopathy: an update meta-analysis. Clin Res Hepatol Gastroenterol 2015; 39: 674-82.

Received: 8.02.2021

Accepted: 18.02 .2021 